\title{
A well-posed finite-strain model for thin elastic sheets with bending stiffness
}

\author{
David J. Steigmann \\ Department of Mechanical Engineering \\ University of California \\ Berkeley, CA. 94720 \\ USA
}

Abstract: An accurate, well-posed two-dimensional model incorporating stretching and bending effects, suitable for analyzing the wrinkling pattern in stretched sheets, is derived from three-dimensional nonlinear elasticity theory.

Keywords: Wrinkling, finite elasticity, thin plates.

\section{Introduction}

Koiter's well-known plate theory theory $[1,2]$ furnishes an entirely satisfactory order $-h^{3}$ model ( $h$ is the plate thickness) of combined stretching and bending for isotropic materials. This is derived from three-dimensional elasticity theory for finite deformations with small strains. It is widely regarded as the 'best' model that combines stretching and bending effects [3]. Hilgers and Pipkin [4-6] initiated the study of the relationship between the two- and three-dimensional theories in the case of finite elastic strain. In the course of deriving an order $-h^{3}$ two-dimensional system from the three-dimensional theory, they noted that the resulting model is generally ill-posed as a minimization problem because the operative version of the Legendre-Hadamard necessary condition [7] fails unless the (plane) stress is pointwise non-compressive. This unexpected restriction on stress, which is normally associated with membrane theory [8], implies that energy minimizers generally fail to exist. To offset this undesirable feature, Hilgers and Pipkin supplemented their model with an ad-hoc regularizing term that is unrelated to the three-dimensional parent theory. However, the finding that the basic order - $h^{3}$ model is ill-posed does not constitute a deficiency of the model per se, because deformations that minimize the energy in the three-dimensional theory generally do not minimize finite-order truncations of the energy [9].

Nevertheless an order - $h^{3}$ model that yields a meaningful minimization problem in its own right is clearly desirable from the viewpoints of analysis and application. In particular, the classical linear models are of this kind. They are invariably based on the assumption that the underlying prestress vanishes, avoiding the difficulty that arises in the nonlinear theory. More recently, Steigmann and Ogden [10] have shown that a well-posed model corresponding to classical plate-buckling theory follows from the linear three-dimensional theory of incremental elasticity provided that the prestress associated with the underlying finite deformation scales appropriately with plate thickness, vanishing in the zerothickness limit. This too alleviates the difficulty observed by Hilgers and Pipkin. Here, guided by these observations, we use the basic Hilgers-Pipkin framework, as modified by Steigmann [9,11], to obtain a 
model that is free of the destabilizing term and which is compatible with restrictions imposed by the three-dimensional parent theory for suitably loaded thin bodies. This results in an order $-h^{3}$ model that is well-posed as a minimization problem and of optimal accuracy visa à vis the three-dimensional theory. Our model furnishes the natural extension of Koiter's to finite strain, coinciding with it in the small-strain limit.

Standard notation is used throughout. Bold face is used for vectors and tensors and indices to denote their components. Latin indices take values in $\{1,2,3\}$; Greek in $\{1,2\}$. The latter are associated with in-plane coordinates and associated vector and tensor components. A dot between bold symbols is used to denote the standard inner product. Thus, if $\mathbf{A}_{1}$ and $\mathbf{A}_{2}$ are second-order tensors, then $\mathbf{A}_{1} \cdot \mathbf{A}_{2}=\operatorname{tr}\left(\mathbf{A}_{1} \mathbf{A}_{2}^{t}\right)$, where $\operatorname{tr}(\cdot)$ is the trace and the superscript ${ }^{t}$ is used to denote the transpose. The norm of a tensor $\mathbf{A}$ is $|\mathbf{A}|=\sqrt{\mathbf{A} \cdot \mathbf{A}}$. The linear operator $\operatorname{Sym}(\cdot)$ delivers the symmetric part of its second-order tensor argument; the notation $\otimes$ identifies the standard tensor product of vectors. If $\mathcal{C}$ is a fourth-order tensor, then $\mathcal{C}[\mathbf{A}]$ is the second-order tensor with orthonormal components $\mathcal{C}_{i j k l} A_{k l}$. Finally, we use symbols such as Div and $D$ to denote the three-dimensional divergence and gradient operators, while div and $\nabla$ are reserved for their two-dimensional counterparts. For example, $\operatorname{Div} \mathbf{A}=A_{i j, j} \mathbf{e}_{i}$ and $\operatorname{div} \mathbf{A}=A_{i \alpha, \alpha} \mathbf{e}_{i}$, where $\left\{\mathbf{e}_{i}\right\}$ is a fixed orthonormal basis and subscripts preceded by commas are used to denote partial derivatives with respect to Cartesian coordinates.

Superposed tildes are used to denote three-dimensional fields while variables appearing without the tilde are the restrictions of these fields to a midsurface $\Omega$ embedded in the three-dimensional plate-like body of thickness $h$. The body itself occupies the volume $\kappa=\Omega \times(-h / 2, h / 2)$. Our basic assumption is that the plate is thin in the sense that $h / l \ll 1$, where $l$ is any characteristic length associated with the geometry of $\Omega$. To ease the notation, we adopt $l$ as the unit of length, so that $h \ll 1$, and seek a model for the elastic sheet valid to order $h^{3}$. This is the scaling typically associated with plate bending, and is relevant when the lateral loading on the plate is likewise of order $h^{3}$ after non-dimensionalization.

\section{Three-dimensional theory}

In the purely mechanical setting considered here, the Piola stress of the three-dimensional theory is given by

$$
\tilde{\mathbf{P}}=\mathcal{W}_{\tilde{\mathbf{F}}}
$$

the derivative with respect to the deformation gradient $\tilde{\mathbf{F}}$ of the strain energy, $\mathcal{W}(\tilde{\mathbf{F}})$, per unit reference volume. This is assumed, for the sake of simplicity, not to depend explicitly on the position $\mathbf{x}$ of a material point in the reference $\kappa$. Thus we confine attention to materials with uniform properties. The force per unit area transmitted across a surface in the reference configuration with unit normal $\mathbf{N}$ is

$$
\tilde{\mathbf{t}}=\tilde{\mathbf{P}} \mathbf{N}
$$

It is well known that this, together with the equilibrium equation

$$
\operatorname{Div} \tilde{\mathbf{P}}=\mathbf{0},
$$


are the natural boundary condition and Euler equation for energy-minimizing deformations under conditions of conservative loading without body force, holding on a subset of $\partial \kappa$ and in $\kappa$ respectively.

We seek an expression for the term $E$ in the expansion

$$
\mathcal{E}=E+o\left(h^{3}\right)
$$

of the potential energy

$$
\mathcal{E}=\int_{\kappa} \mathcal{W} d v-\int_{\partial \kappa_{n}} \tilde{\mathbf{t}} \cdot \tilde{\mathbf{y}} d a
$$

under dead-load conditions, say, where $\tilde{\mathbf{y}}(\mathbf{x})$ is the deformation and $\partial \kappa_{n}$ is the part of the boundary where traction is assigned. The result should be of optimal accuracy by the standard of the three-dimensional theory, and it is desirable that it be well-posed in the sense of furnishing a meaningful minimization problem in its own right. The combination of these two features constitutes the contribution of the present work.

Pursuant to our objective, we confine attention to three-dimensional deformations that satisfy the strong-ellipticity condition

$$
\mathbf{a} \otimes \mathbf{b} \cdot \mathcal{M}(\tilde{\mathbf{F}})[\mathbf{a} \otimes \mathbf{b}]>0 \quad \text { for all } \quad \mathbf{a} \otimes \mathbf{b} \neq \mathbf{0},
$$

where

$$
\mathcal{M}(\tilde{\mathbf{F}})=\mathcal{W}_{\tilde{\mathbf{F}} \tilde{\mathbf{F}}}
$$

is the tensor of elastic moduli. It is well known that this condition is necessary for minimizers of the three-dimensional energy. Later, we shall make use of the strain-dependent elastic moduli $\mathcal{C}(\tilde{\mathbf{E}})$, where

$$
\tilde{\mathbf{E}}=\frac{1}{2}\left(\tilde{\mathbf{F}}^{t} \tilde{\mathbf{F}}-\mathbf{I}\right)
$$

is the strain in which $\mathbf{I}$ is the identity for 3 -space, and

$$
\mathcal{C}(\tilde{\mathbf{E}})=\mathcal{U}_{\tilde{\mathbf{E}} \tilde{\mathbf{E}}}
$$

in which

$$
\mathcal{U}(\tilde{\mathbf{E}})=\mathcal{W}(\tilde{\mathbf{F}})
$$

is the associated strain-energy function. An application of the chain rule furnishes the useful connection

$$
\mathcal{M}(\tilde{\mathbf{F}})[\mathbf{A}]=\mathbf{A} \tilde{\mathbf{S}}+\frac{1}{2} \tilde{\mathbf{F}} \mathcal{C}(\tilde{\mathbf{E}})\left[\mathbf{A}^{t} \tilde{\mathbf{F}}+\tilde{\mathbf{F}}^{t} \mathbf{A}\right]
$$

for any tensor $\mathbf{A}$, where

$$
\tilde{\mathbf{S}}=\mathcal{U}_{\tilde{\mathbf{E}}}
$$

is the symmetric second Piola-Kirchhoff stress, given in terms of the Piola stress by

$$
\tilde{\mathbf{P}}=\tilde{\mathbf{F}} \tilde{\mathbf{S}}
$$

We assume $\mathcal{U}(\cdot)$ to be convex in a neighborhood of the origin in strain space, with the origin furnishing an isolated local minimum. Thus $\tilde{\mathbf{S}}$ vanishes at zero strain, and $\mathcal{C}(\mathbf{0})$ is positive definite in the sense that $\mathbf{A} \cdot \mathcal{C}(\mathbf{0})[\mathbf{A}]>0$ for all non-zero symmetric $\mathbf{A}$. Then,

$$
\tilde{\mathbf{S}}=\mathcal{C}(\mathbf{0})[\tilde{\mathbf{E}}]+o(|\tilde{\mathbf{E}}|) .
$$


It follows from (11), (14) and the usual minor symmetries of $\mathcal{C}$ that

$$
\mathcal{M}(\mathbf{I})[\mathbf{A}]=\mathcal{C}(\mathbf{0})[\mathbf{A}]
$$

and hence that our hypotheses yields strong ellipticity at zero strain, a result that is well known in linear elasticity theory. Accordingly, these hypotheses are compatible with (6).

\section{Summary of previous work}

We summarize those aspects of previous work $[9,11]$ that bear directly on the present objective. The parametrization

$$
\mathbf{x}=\mathbf{u}+\varsigma \mathbf{k}
$$

is used to describe the reference placement $\kappa$ of the plate, where $\mathbf{u} \in \Omega, \varsigma \in(-h / 2, h / 2)$ and the origin of position $\mathbf{x}$ lies on the midsurface $\Omega$. The projection

$$
\mathbf{1}=\mathbf{I}-\mathbf{k} \otimes \mathbf{k}
$$

furnishes the identity on the translation space $\Omega^{\prime}$ of $\Omega$. The deformation gradient is then expressible in the form $[9]$

$$
\tilde{\mathbf{F}}=\nabla \tilde{\mathbf{y}}+\tilde{\mathbf{y}}^{\prime} \otimes \mathbf{k}
$$

where $\nabla(\cdot)$ is the gradient with respect to $\mathbf{u}$ at fixed $\varsigma$ and $(\cdot)^{\prime}$ is the partial derivative with respect to $\varsigma$.

The model to be discussed involves the coefficient vectors in the expansion

$$
\tilde{\mathbf{y}}(\mathbf{x})=\mathbf{r}(\mathbf{u})+\varsigma \mathbf{d}(\mathbf{u})+\frac{1}{2} \varsigma^{2} \mathbf{g}(\mathbf{u})+\frac{1}{6} \varsigma^{3} \mathbf{h}(\mathbf{u})+\ldots,
$$

where $\mathbf{r}(\mathbf{u})$ is the position of a material point on the deformed image $\omega$ of the midplane $\Omega$; its gradient $\nabla \mathbf{r}$ maps $\Omega^{\prime}$ to the tangent plane $T_{\omega}$ to $\omega$ at the material point $\mathbf{u}$. The functions $\mathbf{d}(\mathbf{u}), \mathbf{g}(\mathbf{u})$ and $\mathbf{h}(\mathbf{u})$ are the directors. We note that the regularity of the three-dimensional deformation required by the expansion (19) is not implied by Ball's existence theory for equilibria [12]. Nevertheless, any piecewise $C^{2}$ equilibrium deformation, possessing a potential jump in its normal derivative across a smooth surface in $\kappa$, is in fact $C^{2}$ in the presence of strong ellipticity. It is straightforward to show that it is then $C^{n}$ for arbitrary $n$. Further, in [13] strong ellipticity is used with degree-theoretic arguments to obtain partial existence results for classically smooth (i.e., $C^{2}$ ) equilibria. Given our adoption of strong ellipticity it is then reasonable to assume that equilibria are sufficiently smooth to justify (19).

The potential energy of an edge-loaded plate is given to order $h^{3}$ by (see eqs. (111) and (131) of [9])

$$
E=\int_{\Omega} \bar{W} d a+\frac{1}{24} h^{3} \int_{\partial \Omega_{n}} \mathbf{P} 1 \nu \cdot \mathbf{g} d s-L
$$

with

$$
\begin{gathered}
\bar{W}=h \mathcal{W}(\mathbf{F})+\frac{1}{24} h^{3} \mathcal{M}(\mathbf{F})\left[\mathbf{F}^{\prime}\right] \cdot \mathbf{F}^{\prime}, \\
\mathbf{F}=\nabla \mathbf{r}+\mathbf{d} \otimes \mathbf{k}, \quad \mathbf{F}^{\prime}=\nabla \mathbf{d}+\mathbf{g} \otimes \mathbf{k},
\end{gathered}
$$


and

$$
L=\int_{\partial \Omega_{n}}\left(\mathbf{p}_{r} \cdot \mathbf{r}+\mathbf{p}_{d} \cdot \mathbf{d}\right) d s
$$

where

$$
\mathbf{p}_{r}=h \mathbf{t}+\frac{1}{24} h^{3} \mathbf{t}^{\prime \prime}, \quad \mathbf{p}_{d}=\frac{1}{12} h^{3} \mathbf{t}^{\prime} .
$$

We suppose that $\partial \Omega$ is the union of disjoint $\operatorname{arcs} \partial \Omega_{e}$ and $\partial \Omega_{n}$, where essential and natural boundary conditions, respectively, are specified. Here, three-dimensional position is assigned on $\partial \kappa_{e}=\partial \Omega_{e} \times I$, where $I=[-h / 2, h / 2]$, and $\mathbf{t}, \mathbf{t}^{\prime}$ and $\mathbf{t}^{\prime \prime}$ are derived from the assigned $\tilde{\mathbf{t}}$ on $\partial \kappa_{I n}=\partial \Omega_{n} \times I \subset \partial \kappa_{n} ; \boldsymbol{\nu}$ is the exterior unit normal to $\partial \Omega$ lying to the right of $\Omega$ as it is traversed in the sense of Green's theorem. Thus, in principle $\mathbf{p}_{r}$ and $\mathbf{p}_{d}$ are imposed as data on $\partial \Omega_{n}$. However, because of kinematic restrictions on $\mathbf{d}$ arising in the theory, they are assigned in certain combinations rather than individually, as explained in Section 6.

For loading by lateral pressure the plate is fixed along the entire edge $\partial \Omega$ and $\partial \Omega_{n}$ is empty. We suppose a volume of compressible gas to be bounded by the lower lateral surface $\partial \kappa^{-}$of the plate together with the walls of a rigid container. Let $\mathcal{V}^{-}$be the enclosed volume; i.e., the volume of the compressible gas. The pressure-volume relation of the gas is given by the function $p^{-}\left(\mathcal{V}^{-}\right)$. We further assume the upper lateral surface $\partial \kappa^{+}$of the plate to be acted upon by a uniform pressure $p^{+}$of fixed intensity. It is shown in [9] that if $p^{ \pm}=h^{3} P^{ \pm}+o\left(h^{3}\right)$, with $P^{ \pm}$of order unity, then the associated load potential is given to leading order by

$$
L_{p}=h^{3}\left[\int^{V^{-}} P^{-}(x) d x-P^{+} V^{-}\right]
$$

where

$$
V^{-}=\frac{1}{3} \int_{\Omega} \alpha \mathbf{r} \cdot \mathbf{n} d a
$$

in which $\mathbf{n}$ is the unit normal to the deformed midsurface $\omega$ and $\alpha$ is the areal stretch of the midsurface.

In the course of obtaining (20) use has been made of

$$
\operatorname{div}(\mathbf{P} \mathbf{1})+\mathbf{P}^{\prime} \mathbf{k}=\mathbf{0}
$$

which is simply the restriction of the exact eq. (3) to $\Omega$ [9]. Further, Taylor expansions of (2) at the lateral surfaces of the plate, with $\mathbf{N}= \pm \mathbf{k}$ as appropriate, furnish [9]

$$
\mathbf{t}^{+}+\mathbf{t}^{-}=h \mathbf{P}^{\prime} \mathbf{k}+O\left(h^{3}\right) \text { and } \quad \mathbf{t}^{+}-\mathbf{t}^{-}=2 \mathbf{P} \mathbf{k}+O\left(h^{2}\right) .
$$

If $\mathbf{t}^{ \pm}=O\left(h^{3}\right)$, it follows that

$$
\mathbf{P} \mathbf{k}=O\left(h^{2}\right) \quad \text { and } \quad \mathbf{P}^{\prime} \mathbf{k}=O\left(h^{2}\right) .
$$

Order - $h^{3}$ accuracy is then maintained by imposing $\mathbf{P k}=\mathbf{0}$ and $\mathbf{P}^{\prime} \mathbf{k}=\mathbf{0}$ in the coefficients of $h^{3}$ in the expression (20) for the potential energy. The latter conditions are equivalent to [9]

$$
\left\{\mathcal{W}_{\tilde{\mathbf{F}}}(\nabla \mathbf{r}+\mathbf{d} \otimes \mathbf{k})\right\} \mathbf{k}=\mathbf{0} \quad \text { and } \quad\left\{\mathbf{A}_{(\mathbf{k})}(\nabla \mathbf{r}+\mathbf{d} \otimes \mathbf{k})\right\} \mathbf{g}=-\{\mathcal{M}(\nabla \mathbf{r}+\mathbf{d} \otimes \mathbf{k})[\nabla \mathbf{d}]\} \mathbf{k} \text {, }
$$

where $\mathbf{A}_{(\mathbf{k})}$ is the acoustic tensor defined by

$$
\left\{\mathbf{A}_{(\mathbf{k})}(\mathbf{F})\right\} \mathbf{v}=\{\mathcal{M}(\mathbf{F})[\mathbf{v} \otimes \mathbf{k}]\} \mathbf{k}
$$


This is positive definite by virtue of the strong-ellipticity condition (6), implying that $(30)_{2}$ is solvable for $\mathbf{g}$. Strong ellipticity also implies that $(30)_{1}$ is uniquely solvable for $\mathbf{d}[5,6]$. These results may then be used to conclude that the coefficients of $h^{3}$ in (20) are determined by the first and second gradients of $\mathbf{r}(\mathbf{u})$. Regarding the coefficient of $h$, which is the restriction to $\Omega$ of the three-dimensional strainenergy function, strong ellipticity implies that this is minimized, for a given midplane deformation, by the director $\mathbf{d}$ that satisfies $(30)_{1}$. This is proved in [9]. Accordingly, the potential energy is given by (20) in which $\mathbf{d}$ and $\mathbf{g}$ are determined by $(30)_{1,2}$, respectively. We note that $(30)_{1}$ is equivalent to the requirement that the midplane be in a state of plane stress; i.e., that

$$
\mathbf{S k}=\mathbf{0}
$$

Regarding boundary data, we recall that $\tilde{\mathbf{y}}$ is assigned on $\partial \Omega_{e} \times[-h / 2, h / 2]$. This implies that its midsurface value, $\mathbf{r}$, and those of its tangential through-thickness derivatives, $\mathbf{d}$ and $\mathbf{g}$, are assigned on $\partial \Omega_{e}$. However, the latter two fields cannot be assigned arbitrarily if the foregoing model is to apply on the closure of $\Omega$. The assigned values must agree with the continuous extensions to $\partial \Omega_{e}$ of the functions delivered by equations $(30)_{1,2}$. This effectively means that $\mathbf{r}$ and its normal derivative $\mathbf{r}_{, \nu}$ are assigned. For, the midsurface deformation gradient may be decomposed in the form

$$
\nabla \mathbf{r}=\mathbf{r}_{, s} \otimes \boldsymbol{\tau}+\mathbf{r}_{, \nu} \otimes \boldsymbol{\nu}
$$

where $\boldsymbol{\tau}$ and $\boldsymbol{\nu}$ are the unit tangent and normal to the edge and the tangential derivative $\mathbf{r}_{, s}$ is obtained by differentiating $\mathbf{r}$ with respect to arclength on $\partial \Omega_{e}$. The continuous extension to $\partial \Omega_{e}$ of the field $\mathbf{d}$ derived from $(30)_{1}$ is thus controlled by the boundary values of $\mathbf{r}$ and $\mathbf{r}_{, \nu}$. Because the theory does not require the specification of the value of $\mathbf{g}$ on $\partial \Omega_{e}$, we simply view this as the continuous extension of the solution to $(30)_{2}$ to the boundary. If this extension is in conflict with the value of $\mathbf{g}$ derived from the data for the three-dimensional problem, then in principle it is necessary to use three-dimensional theory in a region adjoining the boundary and then match its predictions to those of the present model in the interior. Alternatively, the present theory applies on the closure of $\Omega$ if the conflicting aspects of the three-dimensional data are relaxed.

\section{The present model}

Unfortunately the functional of $\mathbf{r}(\mathbf{u})$ described by (20) and (30) does not satisfy the relevant version of the Legendre-Hadamard necessary condition [7] for energy minimizers unless the associated stress $\mathbf{S}$ is non-negative definite [6]. The restriction arises from the coefficient of $h^{3}$ via (30), and is associated with variational problems in which the integrand depends on the second gradient of the field in question; here, this arises from the dependence of $\bar{W}$ on $\nabla \nabla \mathbf{r}$, and effectively rules out compressive stresses, however small, implying that in general (20) does not furnish a well-posed minimization problem. While this result is immaterial from the standpoint of the three-dimensional theory, it does pose an obstacle to the use of the two-dimensional model. In [6] this is addressed by introducing an ad hoc regularization arranged in such a way as to satisfy the Legendre-Hadamard condition automatically, without a priori restrictions on the stress. In this way well-posedness is restored, albeit at the expense of accuracy. 
Here we use restrictions arising in the three-dimensional theory to argue that the problematic stress appearing in the coefficient of $h^{3}$ may be suppressed with no adverse effect on accuracy. In this way well-posedness is restored in a model based entirely on the three-dimensional theory. To support this position, we note that in classical plate-buckling theory $[10,14]$ the stress scales as $h^{2}$ whenever the deformation is such that the order - $h^{3}$ term in the strain energy is non-trivial; i.e., whenever bending occurs in the absence of transverse forces. Further, when transverse forces scale as $h^{3}$, the exact eqn. (27) and the estimate $(29)_{2}$ yield the conclusion that $\operatorname{div}(\mathbf{P 1})=O\left(h^{2}\right)$. Integration around an arbitrary closed circuit enclosing a simply-connected part of $\Omega$ implies that the integral of $(\nabla \mathbf{r}) \mathbf{S} \boldsymbol{\nu}$ is then of order $h^{2}$ in magnitude, where $\boldsymbol{\nu}$ is the exterior unit normal to the curve. If the deformation gradient is of order unity, then the integral of $\mathbf{S} \boldsymbol{\nu}$ is of order $h^{2}$. This in turn is consistent with the assumption that the stress $\mathbf{S}$ is of order $h^{2}$.

Membrane theory emerges from finite-elasticity theory when the net lateral traction on the plate scales as $h$ [15]. This corresponds to $\mathbf{P}^{\prime} \mathbf{k}$ being of order unity in (27), and the heuristic argument supports the assumption that $\mathbf{S}$ is likewise of order unity, a conclusion which is borne out by the rigorously derived equations of membrane theory [15]. This kind of reasoning suggests that in the problems of interest in the present work, which entail bending in the presence of smaller lateral loads, the stress $\mathbf{S}$ is likely to be less than order unity in magnitude. The most conservative assumption of this kind is that $|\mathbf{S}|=o(1)$ for small $h$; i.e., that $|\mathbf{S}|$ vanishes with thickness, which proves to be sufficient for our purposes. Of course nothing has been proved, and so it is necessary in principle to use the predictions of the resulting model to verify the assumption a posteriori. This state of affairs is nothing new in Mechanics. For example, elasticians are invariably faced with a similar issue when using linear elasticity in lieu of the nonlinear theory.

If $|\mathbf{S}|=o(1)$, as supposed, then the error incurred by imposing $\mathbf{S}=\mathbf{0}$ in the coefficients of $h^{3}$ in (20) affects the energy at order $o\left(h^{3}\right)$. Accordingly, order $-h^{3}$ accuracy is maintained if (20) is replaced by

$$
E=\int_{\Omega} W d a-L
$$

where

$$
W=h \mathcal{W}(\mathbf{F})+\frac{1}{24} h^{3} \mathcal{M}(\mathbf{R})\left[\mathbf{F}^{\prime}\right] \cdot \mathbf{F}^{\prime},
$$

in which $\mathbf{F}^{\prime}$ is determined by $(22)_{2}$ with

$$
\left\{\mathbf{A}_{(\mathbf{k})}(\mathbf{R})\right\} \mathbf{g}=-\{\mathcal{M}(\mathbf{R})[\nabla \mathbf{d}]\} \mathbf{k}
$$

and $\mathbf{R}$ is the rotation factor in the polar decomposition of $\mathbf{F}$. We have invoked the constitutive hypothesis described at the end of Section 2 to conclude that $|\mathbf{E}|=o(1)$ and thus that $\mathbf{F}$ may be replaced by $\mathbf{R}$ in the coefficients of $h^{3}$ with no adverse effect on accuracy. Here $\mathbf{d}$ is computed using $(30)_{1}$ as it stands, but its gradient $\nabla \mathbf{d}$ is evaluated at zero strain in accordance with the restriction on the stress. This is illustrated in the example of Section 6 , where (36) is used in the form

$$
\left\{\mathcal{C}(\mathbf{0})\left[\mathbf{R}^{t} \mathbf{g} \otimes \mathbf{k}\right]\right\} \mathbf{k}=-\left\{\mathcal{C}(\mathbf{0})\left[\mathbf{R}^{t} \nabla \mathbf{d}\right]\right\} \mathbf{k} .
$$

This in turn is a consequence of (31) with

$$
\mathcal{M}(\mathbf{R})[\mathbf{A}]=\mathbf{R C}(\mathbf{0})\left[\mathbf{R}^{t} \mathbf{A}\right],
$$


which follows from (11) and the minor symmetry of $\mathcal{C}$.

Using [11]

$$
\mathcal{M}(\mathbf{R})\left[\mathbf{F}^{\prime}\right] \cdot \mathbf{F}^{\prime}=\mathcal{M}(\mathbf{I})\left[\mathbf{R}^{t} \mathbf{F}^{\prime}\right] \cdot \mathbf{R}^{t} \mathbf{F}^{\prime}
$$

with (38), together with the minor symmetries of $\mathcal{C}$, we arrive at the final form

$$
W=h \mathcal{W}(\mathbf{F})+\frac{1}{24} h^{3} \mathcal{C}(\mathbf{0})\left[\mathbf{R}^{t} \mathbf{F}^{\prime}\right] \cdot \mathbf{R}^{t} \mathbf{F}^{\prime}
$$

of the strain-energy function. This involves $\nabla \mathbf{r}$ in both terms and $\nabla \nabla \mathbf{r}$ in the second term. It is easily verified that the operative Legendre-Hadamard condition [7], which we do not state here, is satisfied without qualification.

\section{Equilibrium equations and boundary conditions}

The Euler equations for (34) are derived in the manner discussed in [4,9]. They are

$$
\operatorname{div} \mathbf{T}+\alpha(\Delta p) \mathbf{n}=\mathbf{0}, \quad \text { or } \quad T_{i \alpha, \alpha}+\alpha(\Delta p) n_{i}=0,
$$

where $\mathbf{T}$ is the tensor with nontrivial components

$$
T_{i \alpha}=N_{i \alpha}-M_{i \alpha \beta, \beta}, \quad \text { where } \quad N_{i \alpha}=\partial W / \partial r_{i, \alpha} \quad \text { and } \quad M_{i \alpha \beta}=\partial W / \partial r_{i, \alpha \beta},
$$

$\Delta p=p^{-}-p^{+}$is the net inflation pressure, and

$$
\alpha \mathbf{n}=\mathbf{F}^{*} \mathbf{k}
$$

in which $\mathbf{F}^{*}$ is the restriction to $\Omega$ of the cofactor of the deformation gradient. This term may be computed directly from the deformation $\mathbf{r}(\mathbf{u})[16]$ : thus,

$$
\alpha \mathbf{n}=\operatorname{div} \mathbf{G}, \quad \text { or } \quad \alpha n_{k}=G_{k \beta, \beta},
$$

where

$$
G_{k \beta}=\frac{1}{2} e_{i j k} e_{\alpha \beta} r_{i, \alpha} r_{j}
$$

in which $e_{i j k}$ and $e_{\alpha \beta}$ respectively are the three- and two-dimensional unit alternators $\left(e_{123}=e_{12}=+1\right)$. Here all components are referred to a standard orthonormal basis as described in the Introduction.

The derivation of (41) makes use of the fact that the variational derivative (denoted by a superposed dot) of the pressure-load potential (25) is [9]

$$
\dot{L}_{p}=\int_{\Omega} \alpha(\Delta p) \mathbf{n} \cdot \dot{\mathbf{r}} d a
$$

where $\Delta p=h^{3}\left(P^{-}-P^{+}\right)$is the leading-order pressure difference across the plate.

The essential boundary conditions on $\partial \Omega_{e}$ entail the specification of the position $\mathbf{r}$ and its normal derivative $\mathbf{r}_{, \nu}$ (cf. Section 3 ). The natural boundary conditions on $\partial \Omega_{n}$ are $[4,9]$

$$
T_{i \alpha} \nu_{\alpha}-\left(M_{i \alpha \beta} \nu_{\alpha} \tau_{\beta}\right)_{s}=f_{i} \quad \text { and } \quad M_{i \alpha \beta} \nu_{\alpha} \nu_{\beta}=c_{i}
$$

where $f_{i}$ and $c_{i}$ are the force and couple per unit length. If $\partial \Omega_{n}$ is non-empty we set $\Delta p=0$ in the Euler equation $(41)_{1}$. If $\partial \Omega_{n}$ is piecewise smooth, with a finite number of points where its unit tangent $\boldsymbol{\tau}$ is 
discontinuous, then the foregoing must be amended to include corner forces $F_{j}=\left[M_{j \alpha \beta} \nu_{\alpha} \tau_{\beta}\right]$, where the bracket identifies the jump of the enclosed quantity occurring as $\partial \Omega_{n}$ is traversed clockwise. This is explained in detail in [4].

The force and couple densities may be expressed in terms of the vectors $\mathbf{p}_{r}$ and $\mathbf{p}_{d}$ of (24), if desired, by first evaluating the variational derivative of the associated load potential. We have

$$
\dot{L}=\int_{\partial \Omega_{n}}\left(\mathbf{p}_{r} \cdot \dot{\mathbf{r}}+\mathbf{p}_{d} \cdot \dot{\mathbf{d}}\right) d s,
$$

in which $\dot{\mathbf{d}}$ is obtained from the variational derivative of $(30)_{1}$; the latter being presumed to hold in all configurations of the plate. Thus,

$$
\{\mathcal{M}(\mathbf{F})[\nabla \dot{\mathbf{r}}+\dot{\mathbf{d}} \otimes \mathbf{k}]\} \mathbf{k}=\mathbf{0} .
$$

Because $\mathbf{p}_{d}=O\left(h^{3}\right)$ (cf. (24)) we put $\mathbf{F}=\mathbf{R}$ for consistency, obtaining

$$
\left\{\mathbf{A}_{(\mathbf{k})}(\mathbf{R})\right\} \dot{\mathbf{d}}=-\{\mathcal{M}(\mathbf{R})[\nabla \dot{\mathbf{r}}]\} \mathbf{k},
$$

which may be simplified to

$$
\left\{\mathcal{C}(\mathbf{0})\left[\mathbf{R}^{t} \dot{\mathbf{d}} \otimes \mathbf{k}\right]\right\} \mathbf{k}=-\left\{\mathcal{C}(\mathbf{0})\left[\mathbf{R}^{t} \nabla \dot{\mathbf{r}}\right]\right\} \mathbf{k} .
$$

This delivers $\dot{\mathbf{d}}$ as a linear function of $\nabla \dot{\mathbf{r}}$, furnishing $\mathbf{p}_{d} \cdot \dot{\mathbf{d}}$ as a linear function of the tangential and normal derivatives $\dot{\mathbf{r}}_{s}$ and $\dot{\mathbf{r}}_{, \nu}$. The term involving the tangential derivative may then be integrated by parts on $\partial \Omega$ and the resulting integrand may be cast in the form $\mathbf{f} \cdot \dot{\mathbf{r}}+\mathbf{c} \cdot \dot{\mathbf{r}}_{, \nu}$. The force and couple densities $\mathbf{f}$ and $\mathbf{c}$ may then be read off in terms of $\mathbf{p}_{r}, \mathbf{p}_{d}$, the material properties and the geometry of $\partial \Omega$. This is discussed further below for isotropic materials.

\section{Reflection symmetry and isotropy}

Plates that exhibit reflection symmetry of the material properties with respect to the midplane are important in applications. They are exemplified by isotropy relative to $\kappa$, which we develop in detail. Thus we consider strain-energy functions which are such that $\mathcal{U}(\mathbf{E})=\mathcal{U}\left(\mathbf{Q}^{t} \mathbf{E} \mathbf{Q}\right)$ with $\mathbf{Q}=\mathbf{I}-2 \mathbf{k} \otimes \mathbf{k}$. This in turn requires that the function $\mathcal{U}^{\prime}\left(E_{i j}\right)=\mathcal{U}\left(E_{k l} \mathbf{e}_{k} \otimes \mathbf{e}_{l}\right)$ be even in $E_{3 \alpha}$ and $E_{\alpha 3}$.

Let $\gamma_{\alpha}=E_{\alpha 3}=E_{3 \alpha}$ be the transverse shear strain, and let $\Gamma\left(\gamma_{\alpha}\right)$ be the function obtained by fixing all components of $\mathbf{E}$ except $\gamma_{\alpha}$ in the strain-energy function. Then,

$$
\partial \Gamma / \partial \gamma_{\alpha}=\mathbf{e}_{\alpha} \cdot\left(\mathcal{U}_{\mathbf{E}}\right) \mathbf{k}
$$

which vanish by (12) and (32). In materials that exhibit reflection symmetry these restrictions are satisfied at $\gamma_{\alpha}=0$ because the strain energy is then an even function of the transverse shears. The corresponding strain is

$$
\mathbf{E}=\boldsymbol{\epsilon}+\frac{1}{2}\left(\varphi^{2}-1\right) \mathbf{k} \otimes \mathbf{k}, \quad \text { where } \quad \boldsymbol{\epsilon}=E_{\alpha \beta} \mathbf{e}_{\alpha} \otimes \mathbf{e}_{\beta}
$$

and $\varphi$ is the transverse stretch. Comparing with the midplane strain obtained from $(22)_{1}$, we conclude that

$$
\mathbf{d}=\varphi \mathbf{n}
$$


where $\mathbf{n}$ is the unit normal to the deformed surface and $\varphi$ is obtained in terms of $\boldsymbol{\epsilon}$ by solving (cf. (32))

$$
\mathbf{k} \cdot \mathbf{S k}=0
$$

That this furnishes a solution to $(30)_{1}$ in the presence of reflection symmetry was proved in $[5,6]$. The uniqueness and energetic optimality of solutions, granted strong ellipticity, were proved in [9, pg. 288]. Therefore reflection symmetry and strong ellipticity, combined with $(30)_{1}$, yield deformations in which the transverse shear strain necessarily vanishes.

Further reduction of the model is developed for isotropic materials. For these we have the well-known representation

$$
\mathcal{C}(\mathbf{0})[\mathbf{A}]=\lambda(\operatorname{tr} \mathbf{A}) \mathbf{I}+2 \mu \operatorname{Sym} \mathbf{A},
$$

where $\lambda$ and $\mu$ are the classical Lamé moduli, satisfying the inequalities $3 \lambda+2 \mu>0$ and $\mu>0$ associated with the positivity of $\mathcal{C}(\mathbf{0})$. This furnishes

$$
\{\mathcal{C}(\mathbf{0})[\mathbf{v} \otimes \mathbf{k}]\} \mathbf{k}=(\lambda+2 \mu) v \mathbf{k}+2 \mu \mathbf{1} \mathbf{v}
$$

for any vector $\mathbf{v}$, where $v=\mathbf{v} \cdot \mathbf{k}$. Using this in (37), with minor effort we derive

$$
\mathbf{k} \cdot \mathbf{R}^{t} \mathbf{g}=-(\lambda+2 \mu)^{-1}\left[\lambda \operatorname{tr}\left(\mathbf{R}^{t} \nabla \mathbf{d}\right)+2 \mu \mathbf{k} \cdot \operatorname{Sym}\left(\mathbf{R}^{t} \nabla \mathbf{d}\right) \mathbf{k}\right] \quad \text { and } \quad \mathbf{1}\left(\mathbf{R}^{t} \mathbf{g}\right)=-2 \mathbf{1}\left\{\operatorname{Sym}\left(\mathbf{R}^{t} \nabla \mathbf{d}\right)\right\} \mathbf{k}
$$

These in turn generate $\mathbf{R}^{t} \mathbf{g}=\mathbf{1}\left(\mathbf{R}^{t} \mathbf{g}\right)+\left(\mathbf{k} \cdot \mathbf{R}^{t} \mathbf{g}\right) \mathbf{k}$ for use in the coefficient of $h^{3}$ in (34), which involves

$$
\nabla \mathbf{d}=\nabla \mathbf{n}+\mathbf{n} \otimes \nabla \varphi
$$

in which $\varphi=1$ has been imposed a posteriori for consistency. We have [11]

$$
\mathbf{R}^{t} \nabla \mathbf{n}=\boldsymbol{\kappa}, \quad \text { with } \quad \boldsymbol{\kappa}=-(\nabla \mathbf{r})^{t} \mathbf{b}(\nabla \mathbf{r})
$$

where $\mathbf{b}$ is the curvature tensor on the deformed surface. In terms of Cartesian coordinates,

$$
\boldsymbol{\kappa}=-b_{\alpha \beta} \mathbf{e}_{\alpha} \otimes \mathbf{e}_{\beta} ; \quad b_{\alpha \beta}=n_{i} r_{i, \alpha \beta}
$$

From $\mathbf{R}^{t} \mathbf{n}=\mathbf{k}$ it follows that

$$
\mathbf{R}^{t} \nabla \mathbf{d}=\boldsymbol{\kappa}+\mathbf{k} \otimes \nabla \varphi \quad \text { and } \quad \mathbf{R}^{t} \mathbf{g}=-\frac{\lambda}{\lambda+2 \mu}(\operatorname{tr} \boldsymbol{\kappa}) \mathbf{k}-\nabla \varphi
$$

and $(22)_{2}$ yields

$$
\mathbf{R}^{t} \mathbf{F}^{\prime}=\mathbf{B}+\mathbf{k} \otimes \nabla \varphi-\nabla \varphi \otimes \mathbf{k}
$$

where

$$
\mathbf{B}=\boldsymbol{\kappa}-\frac{\lambda}{\lambda+2 \mu}(\operatorname{tr} \boldsymbol{\kappa}) \mathbf{k} \otimes \mathbf{k}
$$

Eq. (40) then gives the classical bending energy

$$
\mathcal{C}(\mathbf{0})\left[\mathbf{R}^{t} \mathbf{F}^{\prime}\right] \cdot \mathbf{R}^{t} \mathbf{F}^{\prime}=\mathcal{C}(\mathbf{0})[\mathbf{B}] \cdot \mathbf{B}=\frac{2 \lambda \mu}{\lambda+2 \mu}(\operatorname{tr} \boldsymbol{\kappa})^{2}+2 \mu|\boldsymbol{\kappa}|^{2} .
$$

This energy agrees precisely with the bending energy obtained by the method of gamma convergence [17]. Application of the latter method to derive this result requires that the midplane strain, and the 
associated membrane energy (the leading term in (35)), vanish together. If this strain is non-zero, then the leading-order model is membrane theory. However, the membrane energy given here does not agree with that derived via gamma convergence [18]. Agreement with the gamma limit is secured if the present membrane energy is relaxed in the manner described in [19] (see also [20]). This relaxation entails wrinkling on an arbitrarily fine scale, and provides the conceptual foundation for tension-field theory [21]. Relaxation is not appropriate in the present model, however, because the bending energy penalizes fine-scale wrinkling, allowing the theory to be used to resolve the amplitude and wavelength of the wrinkles. Thus the present model accommodates combined bending and stretching and incorporates the two limit models.

In a study aimed at resolving the details of wrinkling patterns, Cerda and Mahadevan [22] based their analysis on Föppl-von Karmann plate theory (see also [23]). However, the latter theory is known to emerge as the rigorous leading-order approximation to the three-dimensional theory when the potential energy scales as $h^{4}$, in response to commensurate lateral loads, and even then only when the deformation of the midplane is an isometry [17]. The status of [22] via a vis three-dimensional elasticity would thus appear to be open to question to the extent that it purports to accommodate significant stretching.

Regarding the response functions $N_{i \alpha}$ and $M_{i \alpha \beta}$ occurring in (42), we observe that $W$ involves $\nabla \nabla \mathbf{r}$ via $\boldsymbol{\kappa}$. Using (61) and (65) in (42), we derive

$$
M_{i \alpha \beta}=\frac{1}{12} h^{3} n_{i}\left(\frac{2 \lambda \mu}{\lambda+2 \mu} b_{\gamma \gamma} \delta_{\alpha \beta}+2 \mu b_{\alpha \beta}\right) .
$$

On the other hand, $\nabla \mathbf{r}$ is involved in the membrane energy and also in $\boldsymbol{\kappa}$, via the normal $\mathbf{n}$. Using (44) and (45) to evaluate the associated derivative (see [24] for a detailed calculation), we obtain

$$
N_{i \alpha}=h \partial \mathcal{W} / \partial r_{i, \alpha}-M_{i \lambda \mu} \Gamma_{\alpha \lambda \mu}
$$

where $\Gamma_{\alpha \lambda \mu}$ are the Christoffel symbols induced by the parametrization of the deformed surface in terms of the coordinates $u_{\alpha}$. It is well known that the Christoffel symbols depend on the surface metric $r_{i, \alpha} r_{i, \beta}$ and its coordinate derivatives. Explicitly (cf. $\left.(53)_{2}\right)$,

$$
\Gamma_{\alpha \lambda \mu}=E_{\mu \alpha, \lambda}+E_{\alpha \lambda, \mu}-E_{\lambda \mu, \alpha}
$$

where $E_{\alpha \beta}=\frac{1}{2}\left(r_{i, \alpha} r_{i, \beta}-\delta_{\alpha \beta}\right)$, in which $\delta$ is the Kronecker delta, and the strain itself has been neglected in the computation of $\Gamma_{\alpha \lambda \mu}$ to ensure consistency with (35). Accordingly, strain-gradient effects enter the model via the $\Gamma_{\alpha \beta}^{\lambda}$, whereas bending effects are of course accounted for by the $b_{\alpha \beta}$. In applications these typically scale differently [2]. We observe that the associated term in (67) is inherently nonlinear and so does not appear in the linearization of the model with respect to the midplane displacement field $\mathbf{r}(\mathbf{u})-\mathbf{u}$.

The theory calls for the specification of the force and couple densities $\mathbf{f}$ and $\mathbf{c}$ on $\partial \Omega_{n}$ (cf. (47)). Though it is not necessary to relate these to $\mathbf{p}_{r}$ and $\mathbf{p}_{d}$ for the purpose of addressing a particular boundary-value problem, it may nevertheless be desirable to do so for the purpose of interpreting a solution in terms of the three-dimensional theory. To effect this we proceed as above, using (51) to obtain

$$
\mathbf{k} \cdot \mathbf{R}^{t} \dot{\mathbf{d}}=-(\lambda+2 \mu)^{-1}\left[\lambda t r\left(\mathbf{R}^{t} \nabla \dot{\mathbf{r}}\right)+2 \mu \mathbf{k} \cdot \operatorname{Sym}\left(\mathbf{R}^{t} \nabla \dot{\mathbf{r}}\right) \mathbf{k}\right] \quad \text { and } \quad \mathbf{1}\left(\mathbf{R}^{t} \dot{\mathbf{d}}\right)=-2 \mathbf{1}\left\{\operatorname{Sym}\left(\mathbf{R}^{t} \nabla \dot{\mathbf{r}}\right)\right\} \mathbf{k}
$$


This furnishes $\dot{\mathbf{d}}$ as a linear function of $\nabla \dot{\mathbf{r}}$, as claimed in Section 5 , and the procedure described there may be implemented to derive the desired formulas for $\mathbf{f}$ and $\mathbf{c}$. We belabor this point here because the results obtained depend on the deformation of $\partial \Omega_{n}$, which may not be specified a priori in the three-dimensional problem. Thus, although the underlying three-dimensional boundary-value problem is of the dead-load type in which $\mathbf{p}_{r}$ and $\mathbf{p}_{d}$ are prescribed, the derived two-dimensional model is not. Said differently, though

$$
\dot{L}=\int_{\partial \Omega_{n}}\left(\mathbf{p}_{r} \cdot \dot{\mathbf{r}}+\mathbf{p}_{d} \cdot \dot{\mathbf{d}}\right) d s=\int_{\partial \Omega_{n}}\left(\mathbf{f} \cdot \dot{\mathbf{r}}+\mathbf{c} \cdot \dot{\mathbf{r}}_{, \nu}\right) d s
$$

we have

$$
L=\int_{\partial \Omega_{n}}\left(\mathbf{p}_{r} \cdot \mathbf{r}+\mathbf{p}_{d} \cdot \mathbf{d}\right) d s, \quad \text { but } \quad L \neq \int_{\partial \Omega_{n}}\left(\mathbf{f} \cdot \mathbf{r}+\mathbf{c} \cdot \mathbf{r}_{, \nu}\right) d s
$$

modulo an unimportant constant in the equality. The point is underscored by using (47) and (66) to write $\mathbf{c}=M \mathbf{n}$ with $M=n_{i} M_{i \alpha \beta} \nu_{\alpha} \nu_{\beta}$. Then, using [25] $\mathbf{n} \cdot \dot{\mathbf{r}}_{, \nu}=-\overline{\boldsymbol{\tau}} \cdot \boldsymbol{\omega}$, where $\overline{\boldsymbol{\tau}}$ is the unit tangent to the deformed boundary in the sense of Strokes' theorem and $\boldsymbol{\omega}$ is the variation of the surface orientation in the sense that $\dot{\mathbf{n}}=\boldsymbol{\omega} \times \mathbf{n}$, we arrive at $\mathbf{c} \cdot \dot{\mathbf{r}}_{, \nu}=-M \overline{\boldsymbol{\tau}} \cdot \boldsymbol{\omega}$, and thus to the interpretation of $M$ as a pure bending couple along the edge of the deformed plate.

The model is completed by adopting a strain-energy function appropriate for isotropy, specialized to plane stress (cf. (30) 1 ), in the coefficient of $h$ in the strain-energy density (40). This should be such as to 'linearize properly', in accordance with our hypotheses on $\mathcal{C}(\mathbf{0})$, and also satisfy strong ellipticity, at least over a substantial range of strain. Strong ellipticity, in turn, is assured if the strain-energy function is polyconvex [12]. A simple set of criteria ensuring this in the case of isotropy has been given in [26]. These encompass constitutive models studied extensively by Carroll [27].

Acknowledgement: Support of this work by the Powley Fund for Ballistics Research is gratefully acknowledged.

\section{References}

1. W.T. Koiter, 1960, A consistent first approximation in the general theory of thin elastic shells, in: Proc. IUTAM Symposium on the Theory of Thin Elastic Shells, Delft (ed. W.T. Koiter). NorthHolland, Amsterdam, pp. 12-33.

2. W.T. Koiter, 1966, On the nonlinear theory of thin elastic shells. Proc. Knonklijke Nederlandse Akademie van Wetenschappen B69, 1-54.

3. P.G. Ciarlet, 2005, An introduction to differential geometry with applications to elasticity. J. Elasticity 78-79, 3-201.

4. M.G. Hilgers and A.C. Pipkin, 1992, Elastic sheets with bending stiffness. Q. Jl. Mech. Appl. Math. 45, 57-75.

5. M.G. Hilgers and A.C. Pipkin, 1992, Bending energy of highly elastic membranes. Quart. Appl. Math. 50, 389-400.

6. M.G. Hilgers and A.C. Pipkin, 1996, Bending energy of highly elastic membranes II. Quart. Appl. Math. 54, 307-316. 
7. M.G. Hilgers and A.C. Pipkin, 1992, The Graves condition for variational problems of arbitrary order. IMA. J. Appl. Math. 48, 265-269.

8. D.J. Steigmann, 1986, Proof of a conjecture in elastic membrane theory. ASME J. Appl. Mech. 53, 955-956.

9. D.J. Steigmann, 2010, 'Applications of Polyconvexity and Strong Ellipticity to Nonlinear Elasticity and Elastic Plate Theory', in: CISM Course on Applications of Poly-, Quasi-, and Rank-One Convexity in Applied Mechanics, vol. 516 (J. Schröder and P. Neff, eds.). Springer, Wien and New York, pp. 265-299.

10. D.J. Steigmann and R.W. Ogden, Classical plate-buckling theory as the rigorous small-thickness limit of three-dimensional incremental elasticity (manuscript).

11. D.J. Steigmann, 2007, Thin-plate theory for large elastic deformations. Int. J. Non-linear Mech. 42, 233-240.

12. J.M. Ball, 1977, Convexity conditions and existence theorems in nonlinear elasticity. Arch. Ration. Mech. Anal. 63, 337-403.

13. T.J. Healey and P. Rosakis, 1997, Unbounded branches of classical injective solutions to the forced displacement problem in nonlinear elastostatics. J. Elasticity 49, 65-78.

14. A.M.A. van der Heijden, 2009, W.T. Koiter's Elastic Stability of Solids and Structures. Cambridge University Press.

15. D.J. Steigmann, 2009, A concise derivation of membrane theory from three-dimensional nonlinear elasticity. J. Elasticity 97, 97-101.

16. M. Taylor and D.J. Steigmann, 2009, Simulation of laminated thermoelastic membranes. J. Thermal Stresses 32, 448-476.

17. G. Friesecke, R.D. James and S. Müller, 2006, A hierarchy of plate models derived from nonlinear elasticity by gamma-convergence. Arch. Ration. Mech. Anal. 180, 183-236.

18. H. Le Dret and A. Raoult, 1995, The nonlinear membrane model as a variational limit of nonlinear three-dimensional elasticity. J. Math. Pures Appl. 75, 551-580.

19. A.C. Pipkin, 1994, Relaxed energy densities for large deformations of membranes. IMA J. Appl. Math. 52, 297-308.

20. B. Dacarogna, 1989, Direct Methods in the Calculus of Variations. Springer, Berlin.

21. D.J. Steigmann, 1990, Tension-field theory. Proc. R. Soc. Lond. A429, 141-73.

22. E. Cerda and L. Mahadevan, 2003, Geometry and physics of wrinkling. Phys. Rev. Lett. 90, 074302-1 to 4 .

23. E. Puntel, L. Deseri and E. Fried, 2011, Wrinkling of a stretched thin sheet. J. Elasticity 105, $137-170$.

24. D.J. Steigmann and R.W. Ogden, 1999, Elastic surface-substrate interactions. Proc. R. Soc. Lond. A455, 437-74.

25. D.J. Steigmann, 1999, On the relationship between the Cosserat and Kirchhoff-Love theories of elastic shells. Math. Mech. Solids 4, 275-88.

26. D.J. Steigmann, 2003, On isotropic, frame-invariant, polyconvex strain-energy functions. Q.J. 
Mech. Appl. Math. 56, 483-491.

27. M.M. Carroll, 1988, Finite strain solutions in compressible isotropic elasticity. J. Elasticity 20, 65-92. 